\title{
Analysis of the Prevalence and Distribution of Cervical and Thoracic Compressive Lesions of the Spinal Cord in Lumbar Degenerative Disease
}

\author{
Masashi Miyazaki, Toyomi Yoshiiwa, Ryuzo Kodera, Masanori Kawano, Hiroshi Tsumura \\ Department of Orthopaedic Surgery, Faculty of Medicine, Oita University, Oita, Japan
}

\begin{abstract}
Study Design: Retrospective study.
Purpose: The aim of the present study is to analyze the prevalence and distribution of cervical and thoracic compressive lesions of the spinal cord in lumbar degenerative disease, using whole-spine postmyelographic computed tomography.

Overview of Literature: Of the various complications resulting from spinal surgery, unexpected neurological deterioration is the most undesired. There are reports of missed compressive lesions of the spinal cord at the cervical or thoracic level in lumbar degenerative disease.

Methods: There were 145 consecutive patients with symptomatic lumbar degenerative disease evaluated. Before the lumbar surgery, image data were obtained. The following parameters at the cervical and thoracic levels were analyzed: compressive lesions from the anterior parts; compressive lesions from the anterior and posterior parts; ossification of the ligamentum flavum; ossification of the posterior longitudinal ligament; and spinal cord tumor.

Results: Compressive lesions from the anterior parts were observed in 34 cases (23.4\%). Compressive lesions from the anterior and posterior parts were observed in 34 cases $(23.4 \%)$. Lesions of ossification of the ligamentum flavum were observed in 45 cases $(31.0 \%)$. Lesions of ossification of the posterior longitudinal ligament were observed in 15 cases (10.3\%). Spinal cord tumor was not observed. Conclusions: A survey of compressive lesions at the cervical or thoracic level in lumbar degenerative disease is important in preventing unexpected neurological deterioration after the lumbar surgery.
\end{abstract}

Keywords: Cervical spine; Thoracic spine; Neurological deterioration; Lumbar surgery

\section{Introduction}

Of the various complications resulting from spinal surgery, unexpected neurological deterioration is one of the most undesired by both surgeons and patients. The causes of such deterioration have been reported to be hematoma $[1,2]$, nerve root injury [3], and cauda equina syndrome [3-5] at the operative site. Some reports have documented neurological deteriorations resulting from coexisting compressive lesions above the operative site or coexisting upper spinal lesions not identified before the lumbar surgery [6-8].

The increase in the world's elderly population has led to the increase in lumbar degenerative disease and lumbar spine surgery. With aging, degenerative changes can appear in the cervical and thoracic spine [9]. However, there

Received Aug 17, 2012; Revised Sep 28, 2012; Accepted Oct 4, 2012

Corresponding author: Masashi Miyazaki

Department of Orthopaedic Surgery, Faculty of Medicine, Oita University,

1-1 Idaigaoka, Hasama-machi, Yufu-shi, Oita 879-5593, Japan

Tel: +81-97-586-5872, Fax: +81-97-549-6047, E-mail: masashim@oita-u.ac.jp 
are few reports on the compressive lesions of the spinal cord at the cervical or thoracic level in lumbar degenerative disease. In order to avoid unexpected neurological deterioration due to coexisting upper spinal compressive lesions, it is imperative for surgeons to be aware of the prevalence and distribution of the lesions in lumbar degenerative disease.

With advances in imaging technology, thin-slice postmyelographic computed tomography (CT) scans are able to analyze the morphology of the whole spinal canal and spinal cord in detail. The purpose of the present study is to analyze the prevalence and distribution of the cervical and thoracic compressive lesions of the spinal cord in lumbar degenerative disease, using whole-spine postmyelographic CT.

\section{Materials and Methods}

\section{Participants}

Between February 2006 and September 2011, 145 consecutive symptomatic patients had undergone wholespine postmyelographic CT before lumbar surgery at the authors' hospital. The population was comprised of 79 men and 66 women, with the mean age of 69.1 years (range, 29-87 years), with lumbar disk herniation $(n=19)$
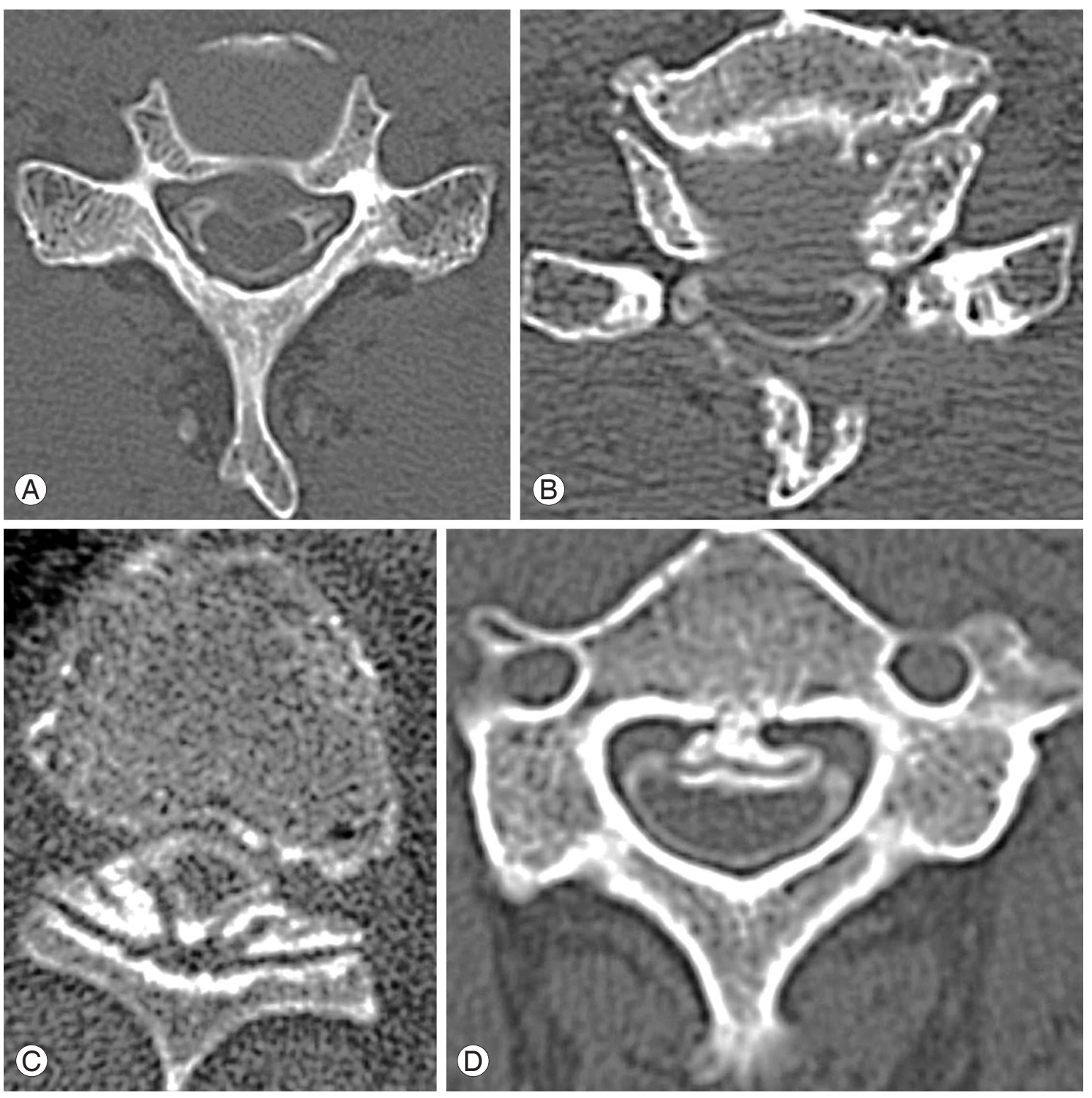

Fig. 1. (A) Compressive lesion from the anterior parts (disc herniation and osteophyte). (B) Compressive lesion from the anterior and posterior parts (hypertrophy of ligamentum flavum). (C) Ossification of the ligamentum flavum. (D) Ossification of the posterior longitudinal ligament. 
or lumbar spine canal stenosis $(\mathrm{n}=126)$. Patients with recent trauma, diagnosis of ossification of the posterior longitudinal ligament or ossification of the ligamentum flavum, rheumatoid arthritis, infectious spondylitis, spinal tumors, or prior fractures were excluded. All patients provided informed consent.

\section{Scanning protocol}

Before the lumbar surgery, the survey of compressive lesions at the cervical and thoracic level in lumbar degenerative disease was performed. Patients were placed in the prone position, with the neck in neutral position. After myelography, CT scans were obtained using a multislice scanner (Toshiba Aquilion 16, Toshiba Medical, Tochigi, Japan). Image data were obtained in $0.5-\mathrm{mm}$ slices, from the level of the occiput to the sacrum.

\section{Image analysis}

Axial images from the cervical to the thoracic level were analyzed. All images were read by two experienced physicians. The following parameters at the cervical and thoracic level were analyzed: compressive lesion from the anterior parts (disc herniation, osteophyte) (Fig. 1A), compressive lesion from the anterior and posterior parts (hypertrophy of ligamentum flavum) (Fig. 1B), ossification of the ligamentum flavum (Fig. 1C), ossification of the posterior longitudinal ligament (Fig. 1D), and spinal cord tumor. We defined compressive lesion of the spinal cord as a lesion in contact with the spinal cord and the anterior or posterior parts, or the morphological deformity of the spinal cord. We defined ossification of the ligamentum flavum and ossification of the posterior longitudinal ligament as an ossified mass arising from the lamina, facet joints, or vertebrae. We calculated the number of affected cases as well as the number of compressive or ossified lesions and the spinal level.

Two independent observers checked each parameter in consensus on the DICOM viewer. In the present study, we investigated the reliability of each parameter in 30 cases in the blinded-method. The reliability of the image evaluation was estimated using the agreement percentage and $\mathrm{K}$ statistics. The agreement was rated as follows: poor, $\mathrm{K}=0$ to 0.2 ; fair, $\mathrm{K}=0.21$ to 0.4 ; moderate, $\mathrm{K}=0.41$ to 0.60 ; substantial, $\mathrm{K}=0.61$ to 0.8 ; and excellent, $\mathrm{K}>0.81$. A value of 1 indicated absolute agreement, whereas a value of 0 indicated agreement no better than chance. The intra- and interobserver agreements were calculated using SPSS ver. 13 (SPSS Inc., Chicago, IL, USA). The intraand interobserver agreement was excellent $(K>0.80)$ in the present study.

\section{Results}

Cervical and thoracic compressive lesions of the spinal

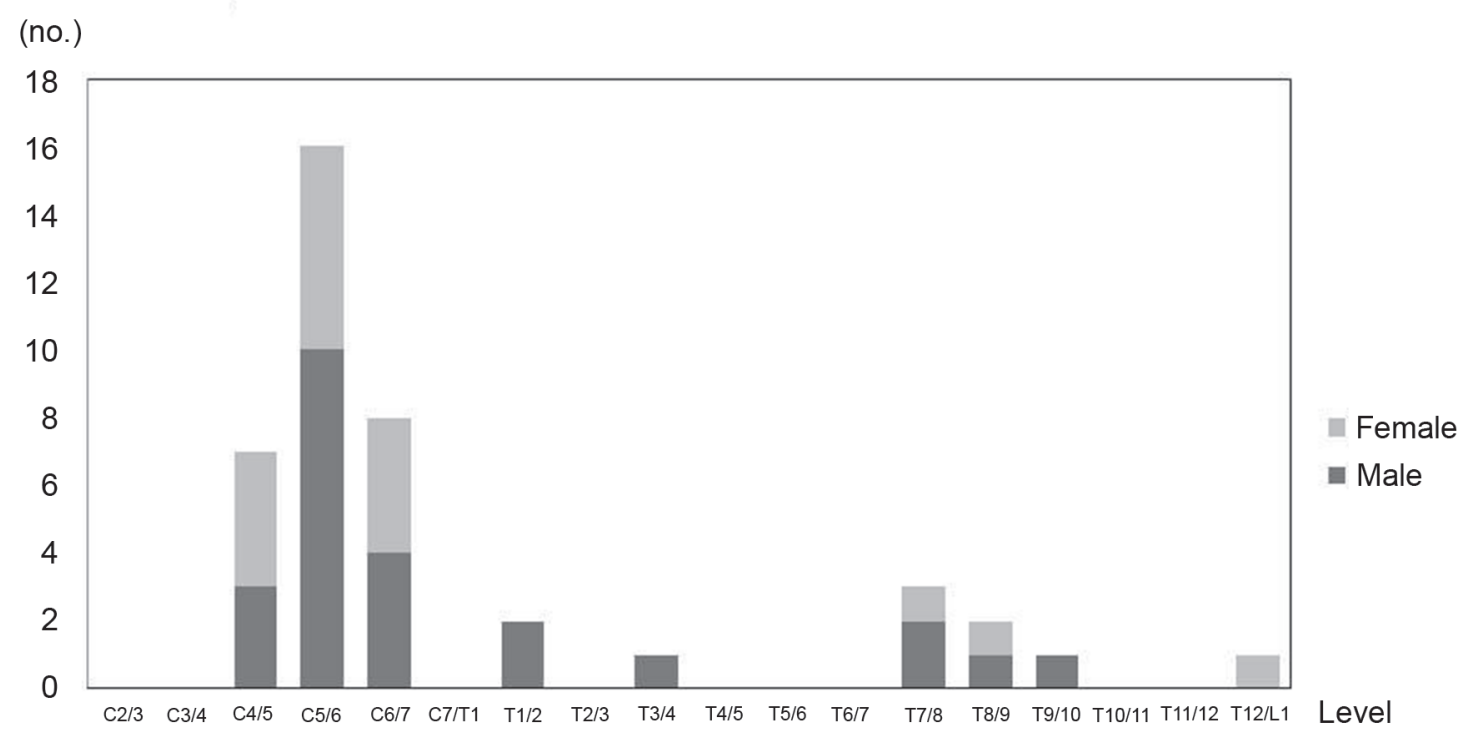

Fig. 2. Sex distribution of the compressive lesions from anterior parts. 
cord from various causes and ossified lesions of the cervical and thoracic spine in lumbar degenerative disease were observed in 89 of 145 patients (61.4\%).

\section{Compressive lesions from the anterior parts}

Compressive lesions from the anterior parts were observed in 34 of 145 patients (23.4\%). The distribution is shown in Fig. 2. The lesions were mainly located at the C5-6 level (16 lesions), followed by the C6-7 level (8 lesions) and C4-5 level (7 lesions). The peak was observed at the middle cervical level, and 1 to 3 lesions were observed at the proximal and middle thoracic level.

\section{Compressive lesions from the anterior and posterior parts}

Compressive lesions from the anterior and posterior parts were observed in 34 of 145 patients (23.4\%). The distribution is shown in Fig. 3. The lesions were mainly located at the C5-6 level (25 lesions), followed by the C4-5 level (13 lesions), C6-7 level (8 lesions), and C3-4 level (7 lesions).
The peak was observed at the middle cervical level, and 1 or 2 lesions were observed at the lower thoracic level.

\section{Lesions of ossification of the ligamentum flavum}

Lesions of ossification of the ligamentum flavum were observed in 45 of 145 patients (31.0\%). The distribution is shown in Fig. 4. The lesions were mainly located at the T11-12 level (18 lesions), followed by the T10-11 level (16 lesions), T9-10 level (11 lesions), and T12-L1 level (8 lesions). One peak was observed at the lower thoracic level, and another small peak was observed at the proximal thoracic level. Six lesions were observed at the T4-5 level, followed by the T3-4 level (4 lesions), and T5-6 level (3 lesions).

\section{Lesions of ossification of the posterior longitudinal ligament}

Lesions of ossification of the posterior longitudinal ligament were observed in 15 of 145 patients (10.3\%). The distribution is shown in Fig. 5. The lesions were mainly

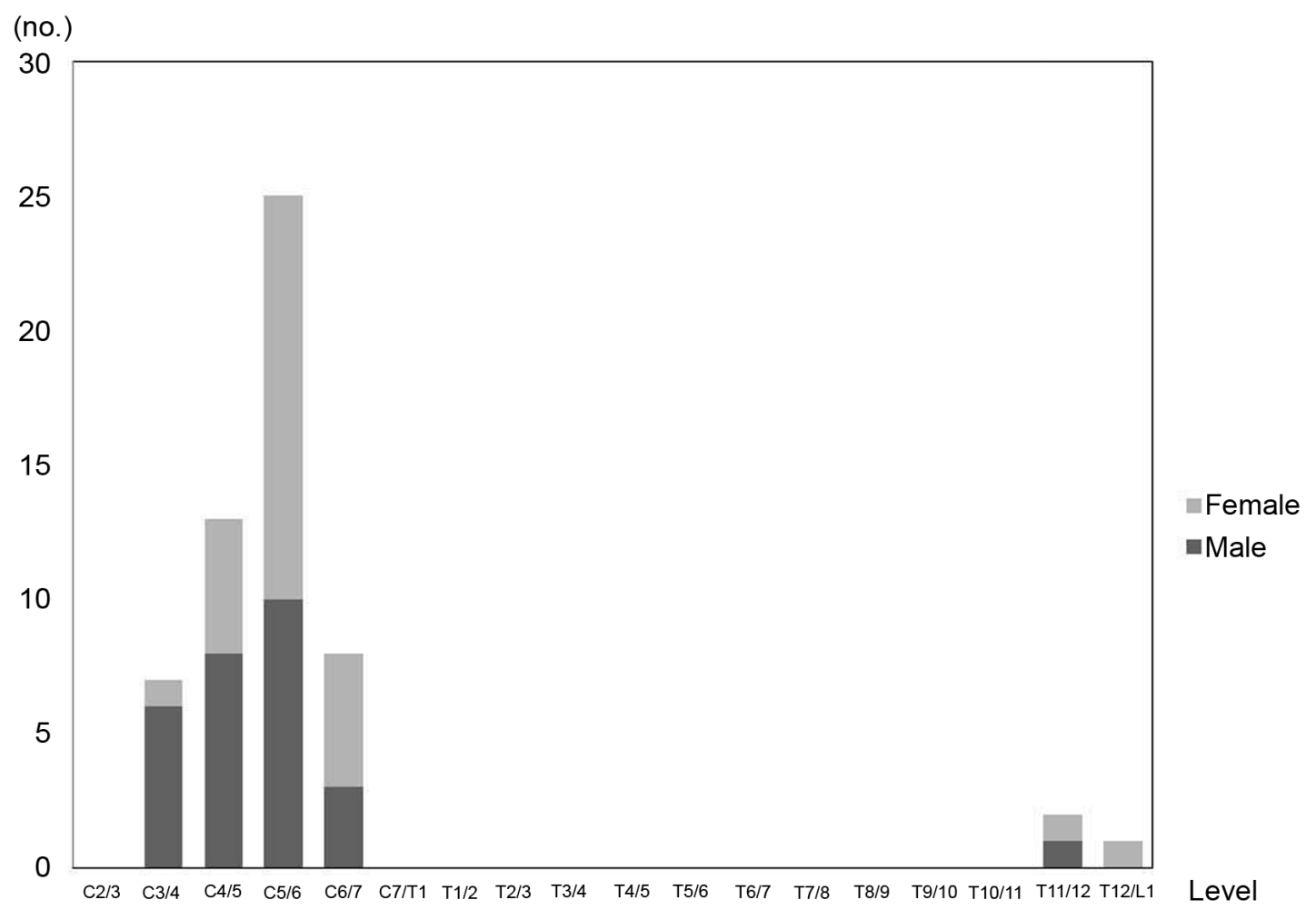

Fig. 3. Sex distribution of the compressive lesions from anterior and posterior parts. 
located at the C5 level (9 lesions), followed by the C4 level (6 lesions). A peak was observed at the middle cervical level, and 1 or 2 lesions were observed at the middle thoracic level.

\section{Spinal cord tumor}

Spinal cord tumor was not observed at the cervical or thoracic level in the present study.

\section{Case reports}

1) Case 1

A 77-year-old woman suffering from lower back pain and an insidious onset of right leg pain was admitted to our hospital for lumbar surgery after her symptoms were aggravated despite conservative treatment. She exhibited no motor weakness. Her biceps and brachioradialis tendon reflexes were normal, but her triceps, patellar, and Achilles tendon reflexes were slightly hypertonic.

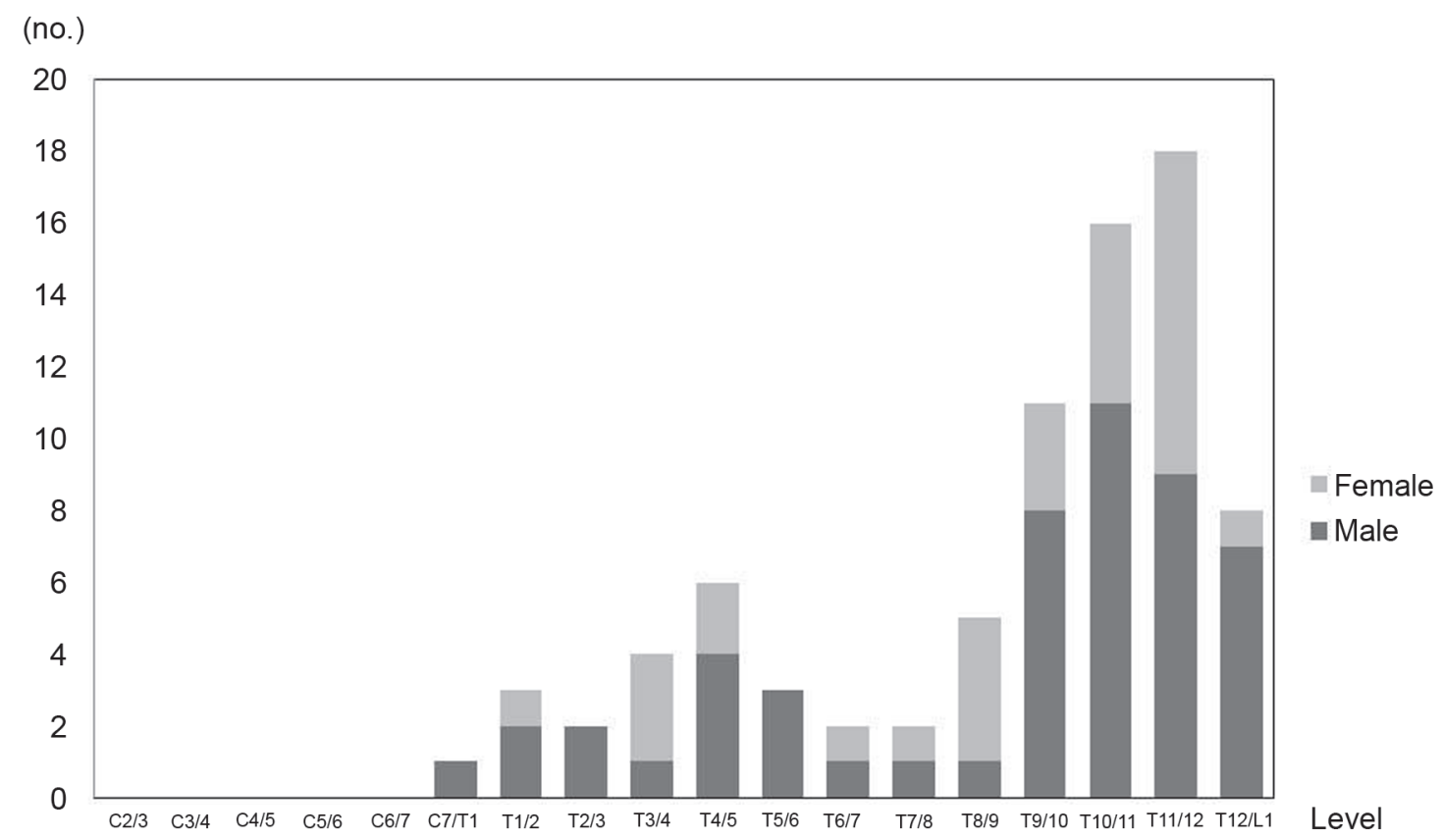

Fig. 4. Sex distribution of the ossification of the ligamentum flavum.

(no.)

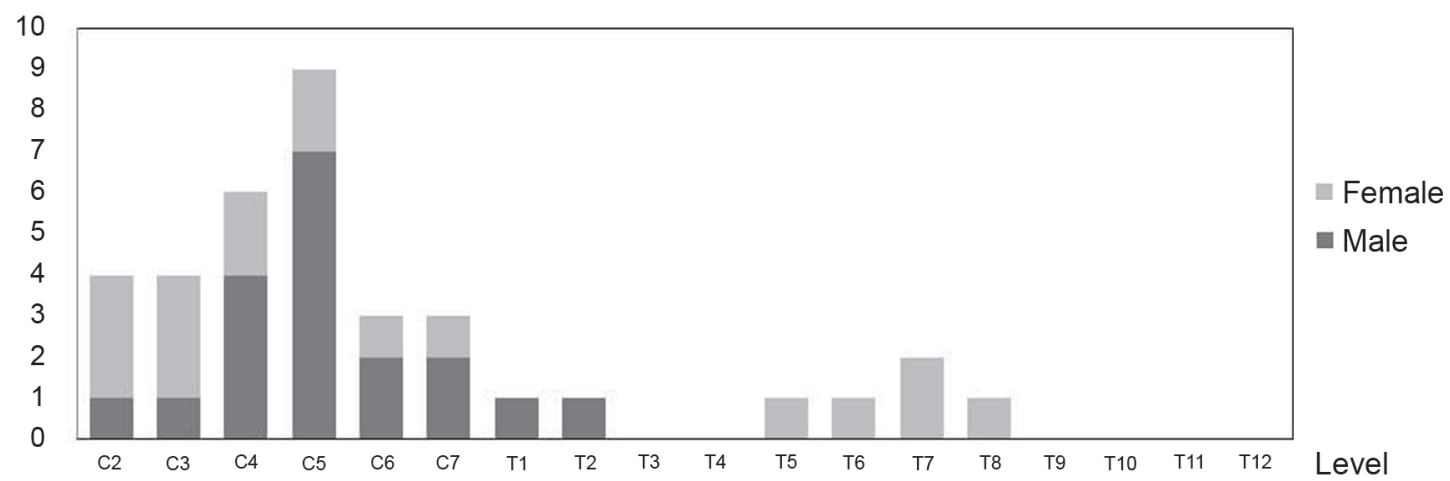

Fig. 5. Sex distribution of the ossification of the posterior longitudinal ligament. 
Magnetic resonance imaging (MRI) of the lumbar spine revealed stenotic changes at the L3-4 level. Preoperative whole spine postmyelographic CT was performed, which revealed stenotic change at the L3-4 level, the right hypertrophic ligamentum flavum compressing the dural sac posteriorly (Fig. 6A), severe stenotic change at the $\mathrm{C} 4-5$ level, and ossification of the posterior longitudinal ligament (Fig. 6B). We provided her with the information on the compressive lesion in the lumbar and cervical spine.

Before decompressive surgery of the L3-4 level, the patient was moved to the prone position, and her neck was postured carefully in a slightly flexed position in order to prevent neurological deterioration of the cervical spine. After the lumbar surgery, the pain in her leg was relieved, and she exhibited no motor weakness, numbness, or deterioration in her fine motor skills. One year after the lumbar surgery, she reported upper-extremity motor weakness and numbness in both her hands as well as deterioration in her fine motor skills. Two years after the lumbar surgery, her triceps, patellar, and Achilles tendon reflexes were exaggerated. A cervical operation was performed to decompress the cervical spinal cord, and 2 years later, her neurological status was improved.

\section{2) Case 2}

A 69-year-old man suffering from an insidious onset of pain and motor weakness in both legs underwent laminectomy for the diagnosis of lumbar spinal canal steno- sis. After the laminectomies, his neurological symptoms deteriorated gradually, and finally, he became paraplegic and was referred to our hospital in emergency. His patellar tendon reflex was slightly hypertonic, but his Achilles tendon reflex was normal. Whole-spine postmyelographic CT was performed, which revealed stenotic change at the C7-T1 (Fig. 7). In this case, ossification of the liga-

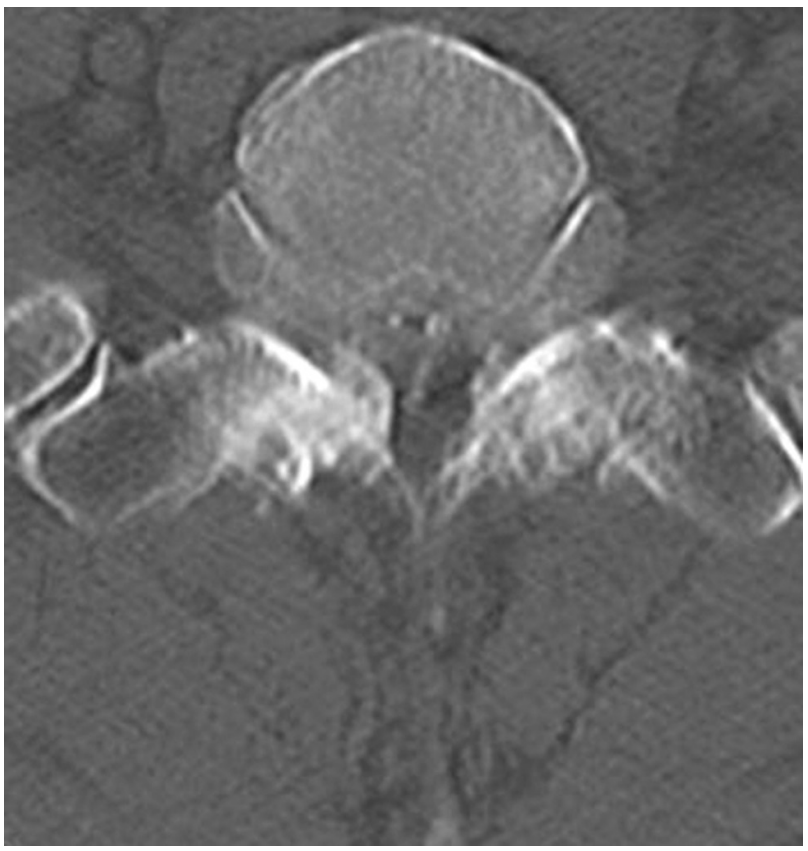

Fig. 7. Case 2. Whole-spine postmyelographic computed tomography scan, demonstrating severe stenotic change at the C7-T1 level and ossification of the ligamentum flavum.
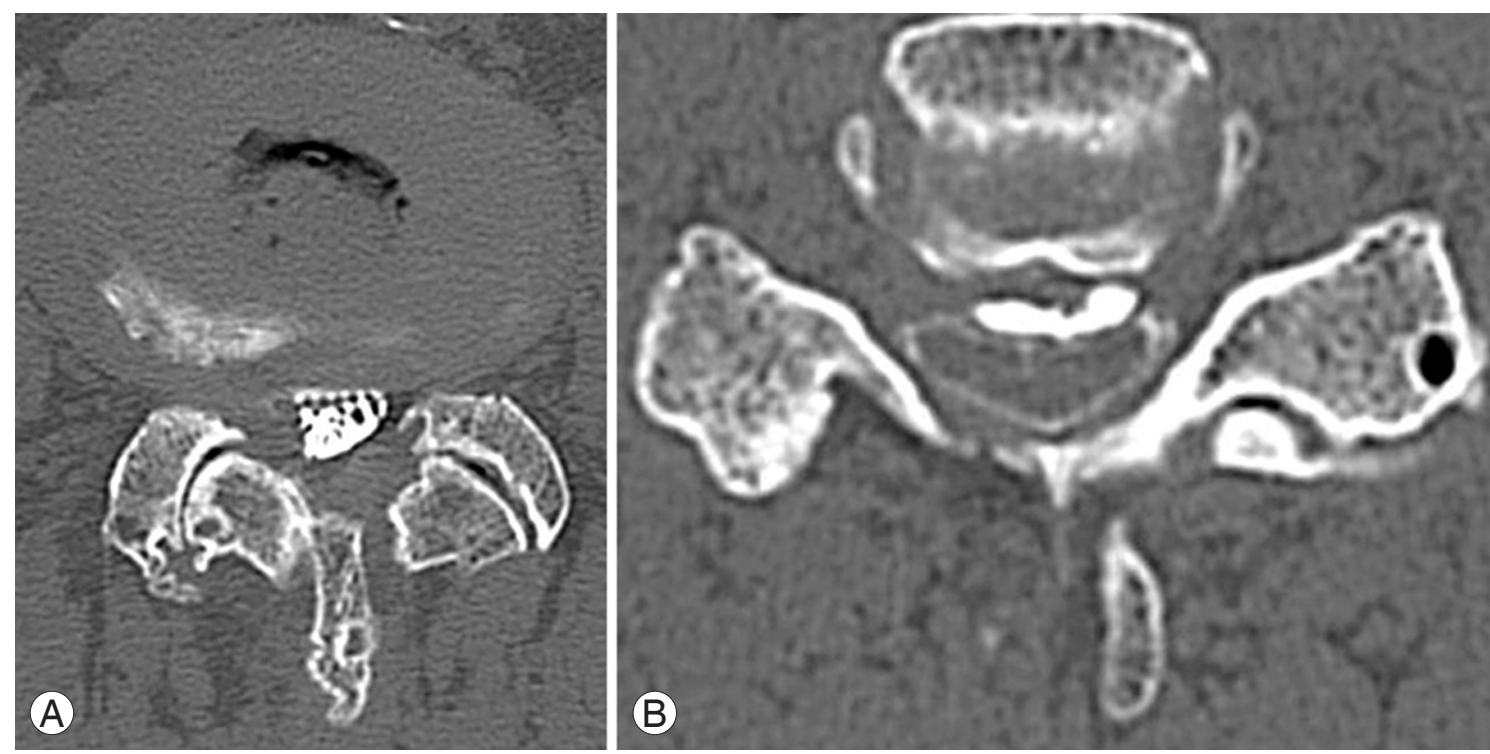

Fig. 6. Case 1. (A) Preoperative postmyelographic computed tomography (CT) scan, demonstrating degenerative spinal canal stenosis at L3-4 and the right hypertrophic ligamentum flavum, compressing the dural sac posteriorly. (B) Preoperative postmyelographic CT scan, demonstrating severe stenotic change at the C4-5 level and ossification of the posterior longitudinal ligament. 
mentum flavum in the cervical-thoracic spine had been missed before the patient underwent lumbar surgery. We performed additional operations to decompress the cervical-thoracic lesion, and his symptoms were resolved.

\section{Discussion}

In spinal surgery, the appearance of unexpected neurological deterioration after the surgery is the worst situation to be faced by the surgeon and patient. There have been numerous reports of cases involving hematoma $[1,2]$, nerve root injury [3], and cauda equina syndrome [3-5] causing postoperative neurological deterioration at the previous operative site. As these complications may be expected, the surgeons inform the patients and receive consent before the surgery. Neurological deterioration due to disorder at the level above the operative site is unexpected, however, and surgeons are unable to properly inform their patients before the surgery. Consequently, such situation hinders the relationship of mutual trust between the surgeon and patient, and often, impedes consent for further treatment.

There rarely have been reports on neurological deterioration due to missed compressive lesion of the spinal cord at the cervical or thoracic level after lumbar decompressive surgery. Such cases tend to have unsuccessful results, and as such, many are not reported. Boccanera and Laus [4] reported three cases of neurological deterioration that developed after lumbar decompressive surgery, where the missed compressive lesions were in the lumbar spines. These cases were reported in 1987, when MRI was not a common diagnostic tool. Such cases are very unlikely to occur under the current medical standards. Valls et al. [6] described the case of one patient in whom thoracic paraparesis developed after a routine lumbar laminectomy, due to a missed spinal arachnoid cyst. Takeuchi et al. [7] reported three cases of thoracic paraparesis which developed after lumbar surgery due to missed spinal cord tumor, disk herniation, and ossification of the ligamentum flavum. Ko et al. [8] described two cases of paraplegia after lumbar surgery due to a missed thoracic meningioma. In all the above cases, preoperative neurological examination, including motor, sensory, and deep tendon reflex, had not suggested cervical or thoracic compressive lesions. After the lumber surgery, however, deep tendon reflexes were exaggerated, suggesting upper spinal compressive lesion.
In this study, the compressive lesions from the anterior parts and compressive lesions from the anterior and posterior parts were mainly located at the C5-6 level, and the peak was observed at the middle cervical level. In the lesions of ossification of the ligamentum flavum, one peak was observed at the lower thoracic level, and another small peak was observed at the proximal thoracic level. In the lesions of ossification of the posterior longitudinal ligament, the peak was observed at the middle cervical level, mainly affecting the cervical spine. Friedenberg and Miller [9] reported the incidence of cervical degenerative disease in both asymptomatic and symptomatic patients by roentgenography; the C5-6 level was observed with the peak at the middle cervical level in both groups. Guo et al. [10] reported on the distribution of ossification of the ligamentum flavum by MRI, and found that the ossified segments were mainly located at the lower thoracic spine, and also commomly found at the upper thoracic spine. Kalb et al. [11] reported that an estimated $70 \%$ of ossification of the posterior longitudinal ligament cases involved the cervical spine. The present study showed similar trends as the previous reports. However, developmental canal stenosis tends to be found at both the cervical and lumbar spine, and there is a high probability that the patients who develop lumbar degenerative disease also suffer from cervical degenerative disease [12]. We need to be aware of such trends and investigate the corresponding levels in order to identify any compressive lesions at the level above the operative site before the lumbar surgery.

In this study, cervical and thoracic compressive lesions of the spinal cord from various causes and ossified lesions of the cervical and thoracic spine in lumbar degenerative disease were observed in 89 of 145 patients (61.4\%). While spinal surgery is not often considered in nonsymptomatic compressive lesions, such lesions may cause symptoms after the surgery. In posterior spinal surgery, a change in position or maintenance of the prone position over a long-term period can cause symptoms after the surgery. As such lesions may cause symptoms in the near future, it may be useful to provide patients with the information.

The present study has several limitations. As this was a cross-sectional study, we did not demonstrate the relationship between compressive lesions at the level above the operative site and the appearance of symptoms. The lesions may remain non-symptomatic. We need to fur- 
ther develop the present study and analyze the risks of the appearance of symptoms at the level above the operative site in greater detail in the future.

\section{Conclusions}

We reported the prevalence and distribution of cervical and thoracic compressive lesions of the spinal cord in lumbar degenerative disease by using postmyelographic CT. Although the relationship between compressive lesions at the level above the operative site and the appearance of symptoms is not clear, further study of the nonsymptomatic compressive lesions may be important in the prevention of unexpected neurological deterioration after the surgery.

\section{Conflict of Interest}

No potential conflict of interest relevant to this article was reported.

\section{References}

1. Gehri R, Zanetti M, Boos N. Subacute subdural haematoma complicating lumbar microdiscectomy. J Bone Joint Surg Br 2000;82:1042-5.

2. Kou J, Fischgrund J, Biddinger A, Herkowitz H. Risk factors for spinal epidural hematoma after spinal surgery. Spine (Phila Pa 1976) 2002;27:1670-3.

3. Schoenecker PL, Cole HO, Herring JA, Capelli AM, Bradford DS. Cauda equina syndrome after in situ arthrodesis for severe spondylolisthesis at the lumbosacral junction. J Bone Joint Surg Am 1990;72:36977.

4. Boccanera L, Laus M. Cauda equina syndrome following lumbar spinal stenosis surgery. Spine (Phila
Pa 1976) 1987;12:712-5.

5. Turker RJ, Slack C, Regan Q. Thoracic paraplegia after lumbar spinal surgery. J Spinal Disord 1995;8:195200.

6. Valls PL, Naul LG, Kanter SL. Paraplegia after a routine lumbar laminectomy: report of a rare complication and successful management. Neurosurgery 1990;27:638-40.

7. Takeuchi A, Miyamoto K, Hosoe H, Shimizu K. Thoracic paraplegia due to missed thoracic compressive lesions after lumbar spinal decompression surgery: report of three cases. J Neurosurg 2004;100:71-4.

8. Ko SB, Lee SW, Shim JH. Paraplegia due to missed thoracic meningioma after laminotomy for lumbar spinal stenosis: report of two cases. Asian Spine J 2011;5:253-7.

9. Friedenberg ZB, Miller WT. Degenerative disc disease of the cervical spine: a comparative study of asymptomatic and symptomatic patients. J Bone Joint Surg Am 1963;45:1171-8.

10. Guo JJ, Luk KD, Karppinen J, Yang H, Cheung KM. Prevalence, distribution, and morphology of ossification of the ligamentum flavum: a population study of one thousand seven hundred thirty-six magnetic resonance imaging scans. Spine (Phila Pa 1976) 2010;35:51-6.

11. Kalb S, Martirosyan NL, Perez-Orribo L, Kalani MY, Theodore N. Analysis of demographics, risk factors, clinical presentation, and surgical treatment modalities for the ossified posterior longitudinal ligament. Neurosurg Focus 2011;30:E11.

12. Edwards WC, LaRocca SH. The developmental segmental sagittal diameter in combined cervical and lumbar spondylosis. Spine (Phila Pa 1976) 1985;10:42-9. 